\title{
Uremia and Inadequate Oxygen Supply Induce Eryptosis and Intracellular Hypoxia in Red Blood Cells
}

\author{
Gabriela Ferreira Dias ${ }^{a, b} \quad$ Sara Soares Tozoni ${ }^{a} \quad$ Gabriela Bohnen ${ }^{a} \quad N^{2}$ dja Grobe ${ }^{b}$ \\ Silvia D. Rodrigues ${ }^{c}$ Tassiana Meireles ${ }^{c}$ Lia S. Nakao ${ }^{c}$ Roberto Pecoits-Filho ${ }^{a, d}$ \\ Peter Kotanko ${ }^{b, e} \quad$ Andréa Novais Moreno-Amarala

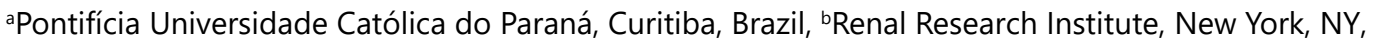 \\ USA, CUniversidade Federal do Paraná, Curitiba, Brazil, dArbor Research Collaborative for Health, Ann \\ Arbor, MI, USA, elcahn School of Medicine at Mount Sinai, New York, NY, USA
}

\section{Key Words}

Eryptosis • Uremic toxins • Chronic kidney disease $\cdot$ Anemia $•$ Hypoxia

\begin{abstract}
Background/Aims: Chronic kidney disease is frequently accompanied by anemia, hypoxemia, and hypoxia. It has become clear that the impaired erythropoietin production and altered iron homeostasis are not the sole causes of renal anemia. Eryptosis is a process of red blood cells (RBC) death, like apoptosis of nucleated cells, characterized by $\mathrm{Ca}^{2+}$ influx and phosphatidylserine (PS) exposure to the outer RBC membrane leaflet. Eryptosis can be induced by uremic toxins and occurs before senescence, thus shortening RBC lifespan and aggravating renal anemia. We aimed to assess eryptosis and intracellular oxygen levels of RBC from hemodialysis patients (HD-RBC) and their response to hypoxia, uremia, and uremic toxins uptake inhibition. Methods: Using flow cytometry, RBC from healthy individuals (CON-RBC) and HD-RBC were subjected to PS (Annexin-V), intracellular $\mathrm{Ca}^{2+}$ (Fluo-3/AM) and intracellular oxygen (Hypoxia Green) measurements, at baseline and after incubation with uremic serum and/or hypoxia $\left(5 \% \mathrm{O}_{2}\right)$, with or without ketoprofen. Baseline levels of uremic toxins were quantified in serum and cytosol by high performance liquid chromatography. Results: Here, we show that HD$\mathrm{RBC}$ have less intracellular oxygen and that it is further decreased post-HD. Also, incubation in $5 \% \mathrm{O}_{2}$ and uremia triggered eryptosis in vitro by exposing PS. Hypoxia itself increased the PS exposure in HD-RBC and CON-RBC, and the addition of uremic serum aggravated it. Furthermore, inhibition of the organic anion transporter 2 with ketoprofen reverted eryptosis and restored the levels of intracellular oxygen. Cytosolic levels of the uremic toxins pCS and IAA were decreased after dialysis. Conclusion: These findings suggest the participation of uremic toxins and hypoxia in the process of eryptosis and intracellular oxygenation.
\end{abstract}




\section{Cellular Physiology Cell Physiol Biochem 2021;55:449-459 \\ \begin{tabular}{ll|l} 
and Biochemistry $\begin{array}{l}\text { DOl: 10.33594/000000396 } \\
\text { Published online: } 14 \text { July 2021 }\end{array}$ & $\begin{array}{l}\text { O } 2021 \text { The Author(s). Published by } \\
\text { Cell Physiol Biochem Press GmbH\&Co. KG }\end{array}$ \\
\cline { 2 - 3 } &
\end{tabular} \\ Ferreira Dias et al.: Hypoxia and Uremia in Eryptosis}

\section{Introduction}

The primary function of human red blood cells (RBC), which comprise approximately $40 \%$ of the blood volume, is the hemoglobin-mediated oxygen transport [1] from the lungs to organs and tissues [2]. The RBC production by the bone marrow is regulated by erythropoietin (Epo), a hormone that maintains an adequate erythropoiesis rate. Anemia is observed in $70 \%$ of the patients undergoing dialysis and is mainly attributed to the lack of Epo production by the diseased kidneys and impaired iron homeostasis [3]. Despite the administration of high doses of erythropoietin stimulating agents (ESA), factors such as inflammation, accumulation of uremic toxins (UT), and reduced RBC lifespan [4] contribute to ESA hypo-responsiveness in 5-10\% of the kidney failure population [5]. The reduced RBC lifespan might occur due to an apoptosis-like RBC death, called eryptosis. This premature $\mathrm{RBC}$ death can be triggered by increased cytosolic $\mathrm{Ca}^{2+}$ content and is initiated through phosphatidylserine (PS) exposure on the outer RBC membrane leaflet [6]. Phagocytic cells recognize PS on the RBC surface and upon receptor-mediated binding remove these cells from circulation. This process may contribute to worsening renal anemia [7, 8]. It is known that hemodialysis (HD) patients have accentuated eryptosis compared to healthy individuals $[9,10]$. This is attributed, in part, to the action of protein-bound uremic toxins (PBUT), such as indoxyl sulfate (IS) $[10,11]$.

About $10 \%$ of the patients undergoing HD show prolonged intradialytic hypoxemia (PIH), defined as arterial oxygen saturation below 90\%, during at least a third of the dialysis session. PIH is associated with higher all-cause mortality and hospitalization as well as higher doses of ESA administration, indicating some degree of ESA hypo-responsiveness [12]. This clinical observation gave rise to studies of the combined effects of hypoxia and uremic toxins. We found evidence that hypoxia and uremia may increase eryptosis in vitro through an imbalance of the intracellular redox environment [13]. This study aimed to explore the role of hypoxia and uremia in the genesis of eryptosis and to evaluate RBC intracellular oxygen levels in HD patients. We hypothesized that (i) hypoxia and uremia increase eryptosis in RBCs from healthy subjects (CON-RBC) and HD patients (HD-RBC) in vitro; (ii) low oxygen levels are observed in HD-RBC, particularly after dialysis; (iii) increased eryptosis and decreased oxygen levels are partially attributed to the extra- and intracellular accumulation of PBUT.

\section{Materials and Methods}

\section{Subjects and blood sampling}

The study was approved by the ethics committee of Pontifícia Universidade Católica do Paraná (registration number 1.752.213). Participants gave informant consent before blood collection. Demographic and biochemical data were collected from patients' medical records or certified laboratory results for healthy subjects. Healthy subjects were required to have no history of renal or inflammatory disease and to have not received anti-inflammatory medication or blood transfusion one month prior to enrollment. All patients were undergoing HD with high-flux dialyzers for at least 3 months prior to enrollment. In hemodialysis patients, blood was drawn pre- and post-HD. In healthy subjects, blood was drawn by venipuncture. Samples were collected in tubes containing 3.2\% sodium citrate and in SST tubes for serum collection. Blood samples were centrifuged (3000 rpm, $15 \mathrm{~min}, 4^{\circ} \mathrm{C}$ ), buffy coat and plasma were discarded. RBCs were washed twice with cold phosphate-buffered saline (PBS) (1500 rpm, $\left.10 \mathrm{~min}, 4^{\circ} \mathrm{C}\right)$ and immediately analyzed.

\section{$R B C$ treatments}

RBCs from HD patients and healthy subjects were incubated for 24 hours under hypoxic conditions in an atmosphere comprising 5\% $\mathrm{O}_{2}$ and 5\% $\mathrm{CO}_{2}$ using the controllers ProOx 110 and ProCO2 120 (Biospherix, Redfield, NY, USA), respectively. Normoxic cells were incubated under physiological conditions, with $21 \% \mathrm{O}_{2}$ and $5 \% \mathrm{CO}_{2}$. The effect of uremia was tested by the addition of $10 \%$ healthy (S-CON) or HD (S-HD) serum to the incubation medium. To each patient, a healthy subject was randomly assigned, forming a patient-donor pair. To increase randomness in the study, before every experiment new patient-donor pairs were selected. 
RBC from healthy donors (CON-RBC) were incubated with autologous serum (S-CON) or S-HD serum from a randomly assigned HD patient. RBC from HD patients (HD-RBC) were incubated with autologous serum (SHD) or healthy serum (S-CON) from a randomly assigned healthy subject (Fig. 1). Serum was diluted with TrisGlucose-BSA buffer (composition (in $\mathrm{mM}$ ): 21.0 tris [hydroxymethyl] aminomethane; $4.7 \mathrm{KCl} ; 2.0 \quad \mathrm{CaCl}_{2}$, $140.5 \mathrm{NaCl} ; 1.2 \mathrm{MgSO}_{4}, 5.5$ glucose and $4 \%$ of bovine serum albumin (Sigma-Aldrich, St. Louis, MO, USA) at $\mathrm{pH}$ 7.4. Additionally, we incubated RBC for 24 hours with or without the addition of $30 \mu \mathrm{M}$ ketoprofen (KETO), an organic anion transport 2 (OAT2) inhibitor [14] to block the entry of UT.

\section{Measurement of PS exposure}

The incubation with Annexin- $V$ PE (BD Bioscience, Sparks, MD, USA) was performed for 15 minutes in the

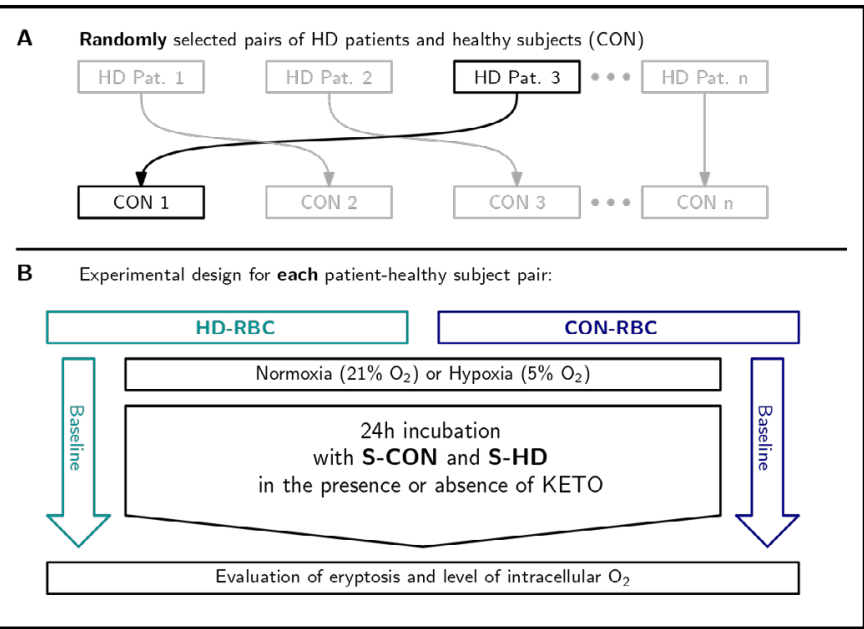

Fig. 1. Schematic overview of RBC incubations. A: each HD patient (HD Pat.) was randomly assigned to a healthy subject (CON), forming a patient-healthy subject pair. B: The HD-RBC and CONRBC were separately incubated with HD serum (S-HD) or healthy serum (S-CON), from within the respective patient-healthy subject pair. In this setting, the incubation occurred for 24 hours with or without ketoprofen (KETO), in $5 \% \mathrm{O}_{2}$ or $21 \% \mathrm{O}_{2}$. Eryptosis and levels of intracellular $\mathrm{O}_{2}$ were evaluated immediately after blood collection (baseline) and after incubation. dark and washed once with PBS. Cells were fixed with $200 \mu \mathrm{l}$ of PBS $4 \%$ formaldehyde (Fix-FACS) and analyzed by flow cytometry (FACSCalibur BD Bioscience, Sparks, MD, USA).

\section{Measurement of intracellular $\mathrm{Ca}^{2+}$}

According to the manufacturer's instructions, erythrocytes were stained with $2 \mu \mathrm{M}$ Fluo-3/AM (Thermo Fisher Scientific, Waltham, MA, USA) in Tris-glucose buffer without BSA. After incubation for 40 min at $37^{\circ} \mathrm{C}$, cells were washed thrice with PBS and resuspended in $200 \mu \mathrm{l}$ Tris-glucose buffer without BSA. $\mathrm{Ca}^{2+}$ dependent fluorescence intensity was measured by flow cytometry (FACSCalibur BD Bioscience, Sparks, MD, USA).

\section{Determination of $\mathrm{O}_{2}$ levels}

RBCs were loaded with Hypoxia Green probe (Thermo Fisher Scientific, Waltham, MA, USA) following manufacturer's instructions. With a reduced intracellular oxygen content, the hypoxia green probe releases rhodamine, emitting fluorescence. Cells were incubated with $1 \mu \mathrm{M}$ of the probe diluted in Tris-glucose buffer without BSA for 2 hours (at $37^{\circ} \mathrm{C}$ in the dark). Then, cells were washed once with PBS, fixed with 200 $\mu \mathrm{l}$ Fix-FACS, and analyzed by flow cytometry (FACSCalibur BD Bioscience, Sparks, MD, USA).

\section{Serum and RBCS concentration of protein-bound uremic toxins}

The PBUT indoxyl sulfate (IS), indole 3-acetic acid (IAA), and p-cresyl sulfate (pCS) were quantified in serum and cells by high-performance liquid chromatography (HPLC) and fluorescence detection, as described by Stockler-Pinto et al. [15] and Rodrigues et al. [16], respectively. The analytical method was developed on a Shimadzu Prominence system and detected by a fluorescence detector (Shimadzu RF-20A). Analytes were separated using a C8 Luna column $150 \times 4.6 \mathrm{~mm}, 5 \mu \mathrm{m}$ (Phenomenex, Torrance, CA, USA). The gradient mobile phase consisted of $50 \mathrm{mM}$ ammonium formate $\mathrm{pH} 3.0$ and methanol, with a linear gradient proportion increasing from 25 to $70 \%(\mathrm{v} / \mathrm{v}$ ) along to the run, at a flow rate of $0.7 \mathrm{~mL} / \mathrm{min}$. The fluorescence wavelengths for IS and IAA were $\lambda$ excitation $=280 \mathrm{~nm} / \lambda$ emission $=383 \mathrm{~nm}$. For pCS it was $\lambda$ excitation $=265 \mathrm{~nm} / \lambda$ emission $=290 \mathrm{~nm}$. The concentration of PBUT in RBCs was normalized by protein content, determined by Bradford assay. 


\begin{tabular}{|c|c|c|}
\hline \multirow{2}{*}{$\begin{array}{l}\text { Cellular Physiology } \\
\text { and Biochemistry }\end{array}$} & \multicolumn{2}{|c|}{ Cell Physiol Biochem 2021;55:449-459 } \\
\hline & $\begin{array}{l}\text { DOI: } 10.33594 / 000000396 \\
\text { Published online: } 14 \text { July } 2021\end{array}$ & $\begin{array}{l}\text { O } 2021 \text { The Author(s). Published by } \\
\text { Cell Physiol Biochem Press GmbH\&Co. KG }\end{array}$ \\
\hline
\end{tabular}

Data analysis

The results are presented as mean \pm SD or median (interquartile range). The statistical software was SPSS Statistics version 20 (Chicago, Ill, USA). The graphs were created using GraphPad Prism 5 (La Jolla, CA, USA). We evaluated the data distribution using the Shapiro-Wilk test. Multiple comparisons between groups were done by one-way ANOVA and post hoc least significant difference test. Paired data were analyzed using paired samples t-test. A p $<0.05$ was considered statistically significant.

\section{Results}

Baseline clinical characteristics of study subjects

The clinical characteristics of the healthy subjects $(\mathrm{n}=14)$ and HD-patients $(n=22)$ are shown in Table 1 . Additional biochemical and treatment parameters from HD-patients are shown in Table 2. HD-patients were older compared to healthy subjects ( $58.1 \pm 18.1$ vs. $34.8 \pm 17.3$ years). Also, we found a significant predominance of male sex in HD patients. The CKD etiology showed a high prevalence of hypertension (40\%) and diabetes type II (30\%). HD patients were anemic (Table 1). Dialysis vintage was $41 \pm 35.6$ months (Table 2).

Baseline characteristics of eryptosis

Our results showed that both markers of eryptosis - PS exposure and intracellular $\mathrm{Ca}^{2+}$ levels - were increased in HD-RBC compared to CON-RBC. The mean of the fluorescence intensity for PS was 12.8 higher on the RBC surface from post-HD compared to pre-HD, while $\mathrm{Ca}^{2+}$ influx demonstrated no difference between pre- and post-HD (Fig. 2).

\section{Intracellular oxygen level}

Intracellular oxygen levels were decreased in RBCs from HD patients pre- and post-HD when compared to CON-RBC. It is shown as an increase in hypoxia level (Fig. 3). Additionally, HD treatment was associated with a significant rise in hypoxia level (mean difference $=7.5$ ) (Fig. 3).

\section{Serum and RBC PBUT concentration}

Serum PBUT was significantly decreased after dialysis but remained high compared to healthy serum (Table 3). Intracellularly, only pCS was detected in CON-RBC. Pre-HD RBCs had a higher concentration of all PBUT compared to control. Additionally, pCS and IAA decreased significantly compared to pre-HD (Table 3).
Table 1. Baseline characteristics of participants. Data expressed as mean \pm SD, or binary variables (frequency). $\mathrm{BMI}=$ Body mass index; $\mathrm{Hb}=$ hemoglobin; $\mathrm{HD}=$ hemodialysis; $\mathrm{NA}=$ not applicable. ${ }^{*} \mathrm{p}<0.05,{ }^{* *} \mathrm{p}<0.01$, *** $\mathrm{p}<0.001$ compared to healthy subjects; ${ }^{a} \mathrm{p}<0.001$ compared to pre-HD. ${ }^{\mathrm{b}}$ Pre-dialysis in HD patients

\begin{tabular}{lcc}
\hline Parameters & Healthy Subjects (n=14) & HD Patients (n=22) \\
\hline Demographics & & \\
Age (years) & $34.8 \pm 17.3$ & $58.1 \pm 18.1^{*}$ \\
Males (\%) & 20 & $73^{*}$ \\
Caucasians (\%) & 100 & 88 \\
BMI (kg/m²) & $23.17 \pm 2.6$ & $25.7 \pm 4.5$ \\
CKD Etiology & & \\
Hypertension \% & $\mathrm{NA}$ & \\
Diabetes type II \% & $\mathrm{NA}$ & 40 \\
Obstructive uropathy \% & $\mathrm{NA}$ & 30 \\
Chronic glomerulonephritis \% & $\mathrm{NA}$ & 5 \\
Polycystic kidney \% & $\mathrm{NA}$ & 5 \\
Pyelonephritis \% & $\mathrm{NA}$ & 5 \\
Uncertain \% & $\mathrm{NA}$ & 5 \\
Biochemical Parameters & & \\
Urea ${ }^{\mathrm{b}}$ (mg/dl) & $22.6 \pm 1.1$ & $109.2 \pm 25.6^{* * *}$ \\
Urea post HD (mg/dl) & $\mathrm{NA}$ & $31.4 \pm 11.8^{\mathrm{a}}$ \\
Creatinine (mg/dl) & $0.8 \pm 0.05$ & $8.1 \pm 2.5^{* * *}$ \\
Hb (g/dl) & $13.7 \pm 0.7$ & $11 \pm 2.2^{* *}$ \\
\hline
\end{tabular}

Table 2. Additional biochemical and treatment parameters in HD patients. PTH = parathyroid hormone; $25(\mathrm{OH}) \mathrm{D}=25$-hydroxyvitamin $\mathrm{D}$; EPO = epoetin alfa

\begin{tabular}{lc}
\hline Parameters & HD-Patients $(\mathrm{n}=22)$ \\
\hline Biochemical Parameters & \\
Serum albumin $(\mathrm{g} / \mathrm{dl})$ & $3.7 \pm 0.3$ \\
Serum iron $(\mu \mathrm{g} / \mathrm{dl})$ & $74 \pm 17.7$ \\
Ferritin $(\mu \mathrm{g} / \mathrm{ml})$ & $327.4 \pm 240.5$ \\
Transferrin saturation $(\%)$ & $31.5 \pm 12.6$ \\
Platelets $\left(\mathrm{x} 10^{3} / \mu \mathrm{l}\right)$ & $235.3 \pm 111.8$ \\
Leucocytes $\left(\mathrm{x} 10^{9} / \mathrm{L}\right)$ & $7 \pm 2.1$ \\
PTH (pg/dl) & $215.9 \pm 136.1$ \\
Serum potassium (mmol/L) & $4.9 \pm 0.6$ \\
Serum phosphorus (mg/dl) & $4.5 \pm 1.1$ \\
Serum calcium (mg/dl) & $9.1 \pm 0.8$ \\
25(OH)D (ng/mL) & $22.8 \pm 11$ \\
Glucose (mg/dL) & $116.6 \pm 29.7$ \\
Treatment Parameters & \\
Time in hemodialysis (months) & $41 \pm 35.6$ \\
Treatment time (minutes) & $216.3 \pm 19.9$ \\
Kt/V & $1.68 \pm 0.26$ \\
Heparin (ml) & $1.24 \pm 0.48$ \\
EPO (units per week) & $7000 \pm 3861$ \\
Intravenous iron (mg per treatment) & $10.4 \pm 3.7$ \\
\hline
\end{tabular}




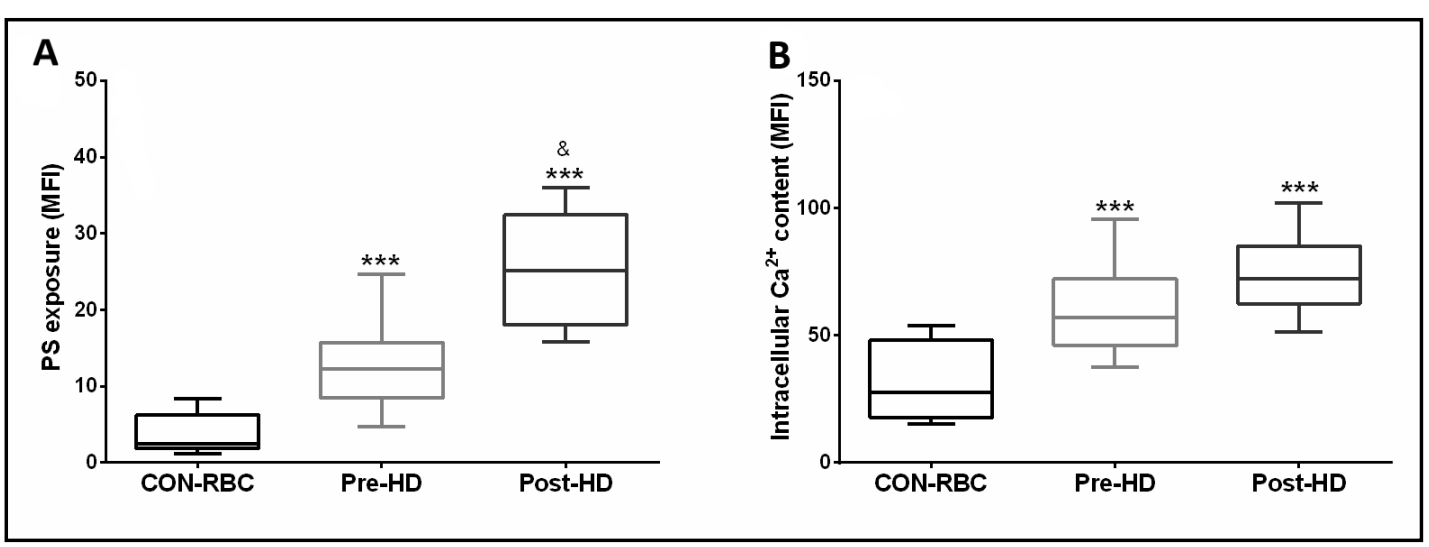

Fig. 2. Levels of eryptosis as indicated by PS exposure (A) and $\mathrm{RBC}$ intracellular $\mathrm{Ca}^{2+}$ content (B). Fresh isolated RBCs from healthy individuals (CON-RBC, $n=12$ ) and HD patients - before (Pre-HD, $n=18$ ) and after (Post-HD, n=12) HD - were loaded with Annexin-V and Fluo-3AM. Data are expressed as mean fluorescence intensity (MFI). ${ }^{* * *} \mathrm{p}<0.001$ compared to CON-RBC. \& means $\mathrm{p}<0.05$ comparing pre vs post HD.

Fig. 3. Hypoxia level in response to HD. Fresh isolated RBCs from healthy individuals (CON-RBC, $\mathrm{n}=6$ ) and ESRD patients - before (Pre-HD, $n=9$ ) and after (Post-HD, n=8) hemodialysis session - were loaded with Hypoxia Green. Data are expressed as mean fluorescence intensity (MFI). ${ }^{* *} \mathrm{p}<0.01 ;{ }^{* * *}$ $\mathrm{p}<0.001$ compared to CON-RBC. ${ }^{\circledR} \mathrm{p}<0.05$ comparing post- vs pre-HD.

\section{Effect of hypoxia on PS exposure}

CON-RBC were exposed to $5 \% \mathrm{O}_{2}$ and HD serum by $24 \mathrm{~h}$ incubation. We observed the $5 \% \quad \mathrm{O}_{2}$ condition itself promoted an increase of PS exposure $(11.3 \%)$ compared to CON-RBC in $21 \% \mathrm{O}_{2}$ ( $4 \pm 1.7 \%)$. In addition, CON-RBC in $21 \%$ $\mathrm{O}_{2}$ incubated with S-HD increased the eryptosis from $4 \pm 1.7$ to $17.6 \pm 6.5 \%$. When both factors - $5 \% \mathrm{O}_{2}$ and S-HD - were combined, eryptosis increased to $25 \%$

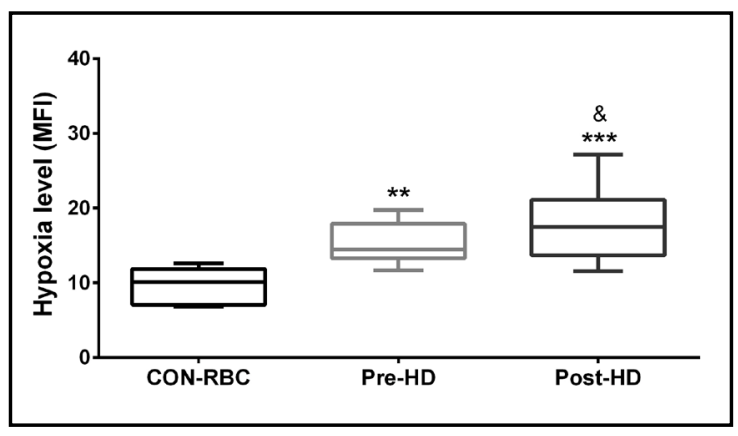

Table 3. PBUT levels in serum and RBC cytoplasm of healthy controls and HD patients before and after hemodialysis

\begin{tabular}{|c|c|c|c|}
\hline PBUT concentration & Control & Pre-HD & Post-HD \\
\hline \multicolumn{4}{|l|}{ In serum } \\
\hline IS $(\mu \mathrm{M})$ & $2.6 \pm 1.8$ & $100.9 \pm 66.9^{* * *}$ & $60.0 \pm 28.8 \& * * *$ \\
\hline $\operatorname{pCS}(\mu \mathrm{M})$ & $15.7 \pm 10.2$ & $243.4 \pm 180.8^{* * *}$ & $143.1 \pm 94.6 \& * * *$ \\
\hline IAA $(\mu \mathrm{M})$ & $1.3 \pm 0.9$ & $12.1 \pm 5.7 * * *$ & $7 \pm 4.3 \& * * *$ \\
\hline \multicolumn{4}{|l|}{ In RBC } \\
\hline IS (pmol/ $/ \mu$ g of protein) & ND & $0.3 \pm 0.4 * *$ & $0.16 \pm 0.17^{* *}$ \\
\hline $\mathrm{pCS}(\mathrm{pmol} / \mu \mathrm{g}$ of protein) & $0.16 \pm 0.12$ & $2.44 \pm 1.89 *$ & $0.42 \pm 0.28 \&$ \\
\hline IAA ( $\mathrm{pmol} / \mu \mathrm{g}$ of protein) & ND & $0.16 \pm 0.18^{* *}$ & ND \& \\
\hline
\end{tabular}
(Fig. 4A). In HD-RBC, the incubation with $5 \% \mathrm{O}_{2}$ promoted a $6.3 \%$ increase of PS exposure compared to HD-RBC in $21 \% \mathrm{O}_{2}$ and healthy serum. S-CON decreased eryptosis of HD-RBC in $21 \% \mathrm{O}_{2}$ (from $27.4 \pm 9.2 \%$ to $16.2 \pm 6 \%$ ) and $5 \% \mathrm{O}_{2}$ (from $37.1 \pm 10.6 \%$ to $22.6 \pm 6.8 \%$ ). The UT inhibitor ketoprofen (KETO) decreased eryptosis in both cell types and treatments, except CON-RBC in $21 \% \mathrm{O}_{2}$ (Fig. $4 \mathrm{~A}$ and B).

\section{Effect of hypoxia on $\mathrm{Ca}^{2+}$ content}

$\mathrm{S}-\mathrm{HD}$ promoted an increase in $\mathrm{Ca}^{2+}$ concentration in CON-RBC, and this effect was amplified in $5 \% \mathrm{O}_{2}$. Surprisingly, KETO increased $\mathrm{Ca}^{2+}$ in CON-RBC incubated in $21 \% \mathrm{O}_{2}$ and had no impact on CON-RBC in $5 \% \mathrm{O}_{2}$. When CON-RBC were incubated with S-HD, KETO reduced $\mathrm{Ca}^{2+}$ concentration in both $21 \% \mathrm{O}_{2}$ and $5 \% \mathrm{O}_{2}$ conditions. The same experimental design was performed using HD-RBC. S-CON diminished intracellular $\mathrm{Ca}^{2+}$ concentrations in normoxic HD-RBC. In HD-RBC, $5 \% \mathrm{O}_{2}$ incubation did not change $\mathrm{Ca}^{2+}$ levels when compared 


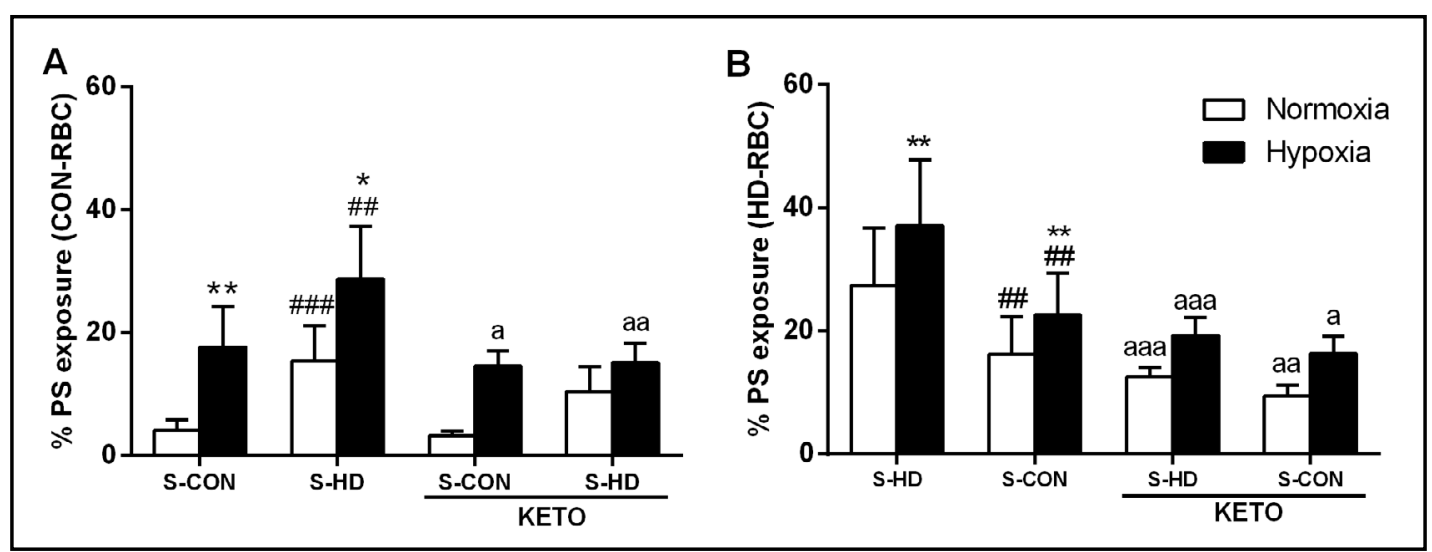

Fig. 4. Phosphatidylserine exposure in response to hypoxia, uremia, and UT entry inhibitor. Isolated RBCs from healthy individuals (CON-RBC, $n=6$ ) and patients pre-HD (Pre/HD-RBC, n=6) were incubated for $24 \mathrm{~h}$ under normoxic $\left(21 \% \mathrm{O}_{2}\right)$ or hypoxic $\left(5 \% \mathrm{O}_{2}\right)$ conditions, in the presence or absence of the UT entry inhibitor ketoprofen (KETO). All treatments contain 10\% serum from the patient-healthy subject pair, which can be pre hemodialysis serum (S-HD) or healthy individuals' serum (S-CON). Data are expressed as mean fluorescence intensity (MFI). ${ }^{* *}$ means $\mathrm{p}<0.01$ and ${ }^{* * *} \mathrm{p}<0.001$ comparing normoxia vs hypoxia; "a" means $\mathrm{p}<0.05$, "aa" $\mathrm{p}<0.01$ and "aaa" $\mathrm{p}<0.001$ comparing KETO group vs no KETO group; \#\# means $\mathrm{p}<0.01$ and \#\#\# $\mathrm{p}<0.001$ comparing autologous serum vs S-HD or S-CON.

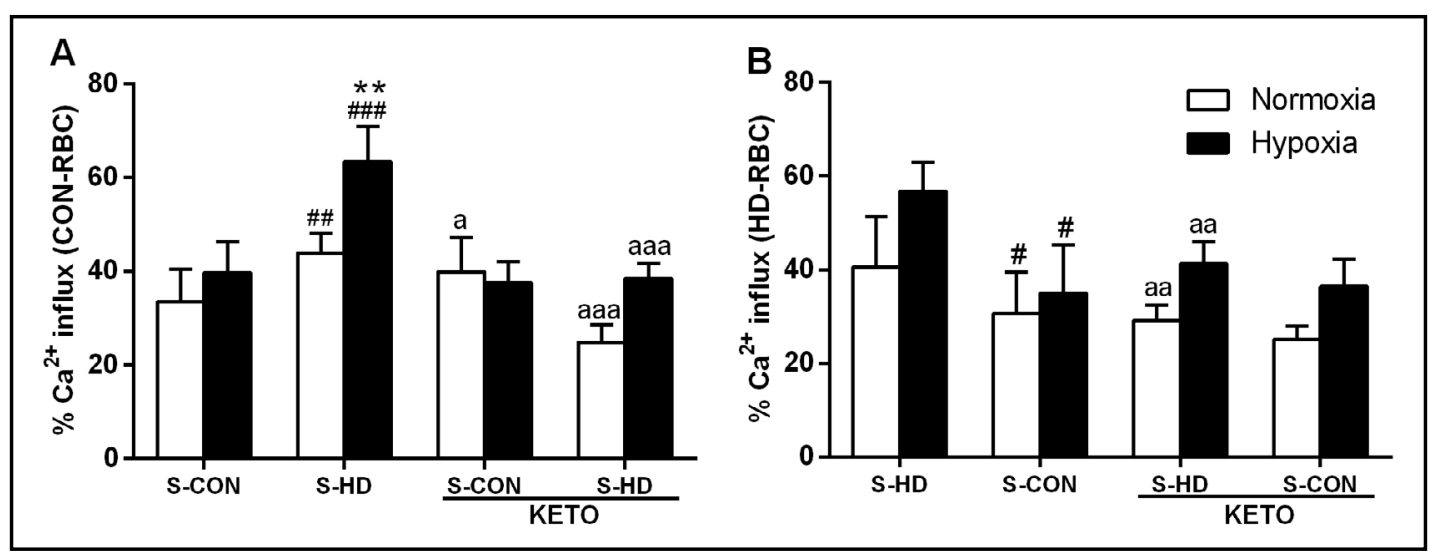

Fig. 5. $\mathrm{Ca}^{2+}$ influx into RBCs in response to uremia, hypoxia, and UT entry inhibitor. Isolated RBCs from healthy individuals (CON-RBC, n=6) and patients pre-HD (Pre/HD-RBC n=6) were incubated for 24h under normoxic $\left(21 \% \mathrm{O}_{2}\right)$ or hypoxic $\left(5 \% \mathrm{O}_{2}\right)$ conditions, in the presence or absence of the UT entry inhibitor ketoprofen (KETO). All treatments contain 10\% serum from the patient-healthy subject pair, which can be pre hemodialysis serum (S-HD) or healthy individuals' serum (S-CON). Data are expressed as mean fluorescence intensity (MFI). ** means $\mathrm{p}<0.01$ comparing normoxia vs hypoxia; "a" means $\mathrm{p}<0.05$, "aa" $\mathrm{p}<0.01$ and "aaa" $\mathrm{p}<0.001$ comparing keto group vs no keto group; \# means $\mathrm{p}<0.05$, \#\# means $\mathrm{p}<0.01$ and \#\#\# $\mathrm{p}<0.001$ comparing autologous serum vs S-HD or S-CON.

to the same treatment in $21 \% \mathrm{O}_{2}$. In the presence of KETO, HD-RBC treated with S-CON presented no difference in $\mathrm{Ca}^{2+}$ levels. However, in HD-RBC incubated with autologous serum, KETO reduced $\mathrm{Ca}^{2+}$ content (Fig. 5).

\section{Effect of uremia on RBC oxygen level}

S-HD per se decreased intracellular oxygen levels in CON-RBC as indicated in Fig. 6A, represented by increased intracellular hypoxia (from $15.28 \pm 1.7$ to $23.49 \pm 2.2$ ). As expected, when incubated in $5 \% \mathrm{O}_{2}$, the increase in CON-RBC intracellular hypoxia was more evident (25 \pm 6.7 ), particularly in the presence of S-HD (41.68 \pm 8 ) (Fig. 6A). The intracellular oxygen 


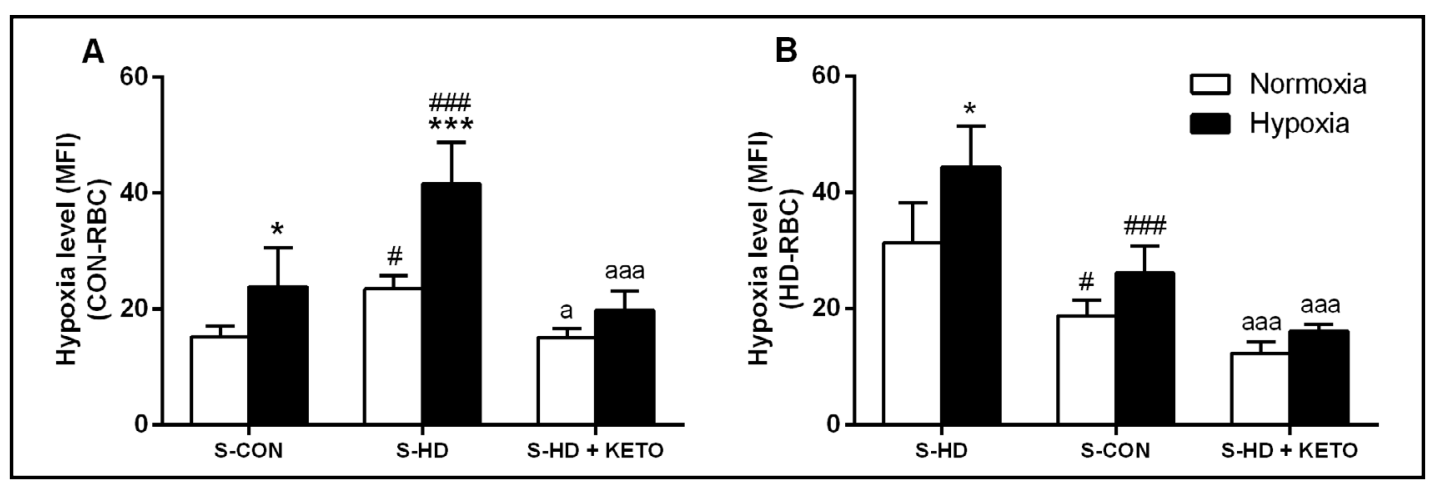

Fig. 6. Intracellular hypoxia level in response to uremia, hypoxia, and UT entry inhibitor. Isolated RBC from healthy individuals (CON-RBC, $n=6$ ) and patients pre hemodialysis (HD-RBC n=6) were incubated for 24h under normoxic $\left(21 \% \mathrm{O}_{2}\right)$ or hypoxic $\left(5 \% \mathrm{O}_{2}\right)$ conditions, in the presence or absence of the UT entry inhibitor ketoprofen (KETO). All treatments contain 10\% serum from the patient-healthy subject pair, which can be pre hemodialysis serum (S-HD) or healthy individuals' serum (S-CON). Data are expressed as mean fluorescence intensity (MFI). * means $\mathrm{p}<0.05,{ }^{* *} \mathrm{p}<0.01$ and ${ }^{* * *} \mathrm{p}<0.001$ comparing normoxia vs hypoxia; "a" means $\mathrm{p}<0.05$ comparing keto group vs no keto group; \#\# means $\mathrm{p}<0.01$ and \#\#\# $\mathrm{p}<0.001$ comparing autologous serum vs S-HD or S-CON.

levels in these cells were restored by the incubations with KETO, both in $21 \% \mathrm{O}_{2}(15 \pm 1.5)$ and $5 \% \mathrm{O}_{2}(19.8 \pm 3.8)$. Additionally, a significant decrease in oxygen levels, and therefore, increased intracellular hypoxia was observed in HD-RBC incubated with S-HD in $21 \% \mathrm{O}_{2}$ $(31.33 \pm 6.8)$ and $5 \% \mathrm{O}_{2}(44.2 \pm 7.0)$. In the presence of $\mathrm{S}-\mathrm{CON}$, the intracellular oxygen levels increased almost $40 \%$, both in $21 \% \mathrm{O}_{2}$ and $5 \% \mathrm{O}_{2}$ conditions (Fig. 6B). It was even more evident in the presence of KETO $(12.3 \pm 1.9 ; 16.1 \pm 1.1$, respectively).

\section{Discussion}

In this study, we demonstrated for the first time that HD-RBC had reduced intracellular oxygen levels compared to CON-RBC, particularly after dialysis. Oxygen levels were restored with the inhibition of OAT2 or absence of UT. Moreover, we found that incubation in 5\% $\mathrm{O}_{2}$ and uremia contribute to the increased eryptosis in RBCs from healthy subjects and HD patients in vitro.

The participation of eryptosis in the context and progression of different pathologies has been studied in the past decades. Eryptosis was observed in clinical conditions such as diabetes type II [17], liver disease [18], hypertension, and dyslipidemia [19]. In accordance with previous findings, our data shows that eryptosis is higher in RBCs from pre-HD patients compared to healthy subjects [10]. PS expression is accentuated after HD, while the increase in $\mathrm{Ca}^{2+}$ levels is observed in pre-HD only [20]. Although it might lead to the interpretation that HD partially regulates $\mathrm{Ca}^{2+}$ concentration and eryptosis would be consequently decreased, PS remains highly exposed.

We showed the incubation in $5 \% \mathrm{O}_{2}$ itself enhanced eryptosis in CON-RBC and HDRBC by exposing PS. In previous studies, S-HD [9] and hypoxia incubation [13] increased PS exposure on healthy RBCs. Here, the highest percentage of PS and $\mathrm{Ca}^{2+}$ levels were observed when both stimuli - S-HD and $5 \% \mathrm{O}_{2}$ - were combined. Interestingly, the incubation in $5 \% \mathrm{O}_{2}$ did not augment $\mathrm{Ca}^{2+}$ concentration either in CON-RBC or HD-RBC, showing that uremia might play a more critical role in this process. However, previous findings indicated that hypoxia increases intracellular $\mathrm{Ca}^{2+}$ content in healthy $\mathrm{RBC}$ incubated in media instead of autologous serum [13]. We interpret that the sustained PS exposure caused by hypoxia might have other triggers, such as the accumulation of UT, or $\mathrm{Ca}^{2+}$ might occur as an early event that is already regulated after 24 hours of incubation. Clinical evidence suggest that acute and moderate hypoxia might have beneficial results, such as the promotion of erythropoiesis. 
On the other hand, chronic and prolonged hypoxemia tend to be pathological [21, 22]. Therefore, studies assessing the effects of hypoxia in different regions of the body are of significant importance. Apart from the main cause of anemia in CKD, which is decreased Epo synthesis and functional iron deficiency [23], we suggest that the increased eryptosis rate and the shortened RBC lifespan [24] reduce the number of circulating RBCs in CKD patients. However, the mechanisms underlying eryptosis in renal anemia and how to correct it are still poorly understood.

In addition to the increased eryptosis by S-HD, isolated UT have been described to trigger eryptosis. IS $[10,11,13]$ and acrolein [25] promoted cell shrinkage and PS exposure. Additionally, IS and indole- 3 acetic acid (IAA) induced a procoagulant phenotype through microparticles release by RBC [26]. Here, we showed an accumulation of IS, pCS, and IAA in HD-RBC pre-dialysis compared to control. Deltombe et al. measured PBUT in RBC, and they found that the rate of transport of the PBUT in pre-HD RBC increases as follows: HA < IS < pCS < IAA. This corroborates our findings, where we observed that only IAA and pCS, not IS, are reduced after HD, indicating different behaviors among these toxins and high transport capacity for pCS and IAA, respectively [27]. As expected, PBUT serum levels were elevated in pre-HD, and although a visible decrease was found after HD, that still significantly high compared to healthy RBC. This is attributed to the PBUT properties, where the binding to albumin disable their clearance by dialysis membranes [28].

We found that the inhibition of the organic anion transporter 2 (OAT2) - a transmembrane RBC protein [29] - by KETO and the incubation with S-CON have the potential to attenuate eryptosis. HD-RBC incubated with S-CON showed a significant decrease in PS exposure. However, it was not comparable to healthy cells, and hypoxia still increased eryptosis in this condition. This evidence suggests the participation of serum components in RBC death. The reduced PS exposure caused by the inhibition of OAT2 indicates the involvement of UT in the process of eryptosis. Our group's previous findings showed an anti-eryptotic effect of KETO in healthy RBCs incubated with IS [10]. Interestingly, KETO reduced PS in non-uremic RBCs in $5 \% \mathrm{O}_{2}$, showing a protective effect against eryptosis promoted by hypoxia. S-CON and KETO acted independently in reducing $\mathrm{Ca}^{2+}$ levels in HD-RBC. Although KETO reduced $\mathrm{Ca}^{2+}$ in CON-RBC incubated with S-HD, it showed an opposite effect by increasing $\mathrm{Ca}^{2+}$ when these cells were in a healthy environment. Decreasing PBUT entry to levels lower than its physiological concentration might be unfavorable since molecules such as IS are known to be beneficial at normal-physiological levels [30].

Herein, we found that RBC from HD patients have low intracellular oxygen levels than healthy RBCs and that this is particularly accentuated post-dialysis. This important finding leads to the assumption that HD-RBC might have disorders in oxygen metabolism either by intrinsic factors, such as the accumulation of UT, or extrinsic factors, such as the extracorporeal circuit. Indeed, our data from 24 hours incubation supports that S-HD promotes a drop in intracellular oxygen levels in CON-RBC in $21 \% \mathrm{O}_{2}$. The increased intracellular oxygen levels in HD-RBC promoted by S-CON and KETO indicate the crucial role of uremic solutes in developing intracellular hypoxemia. In fact, IS induces abnormal oxygen consumption in renal tubular cells and correlates with renal function deterioration [31]. Also, a connection between IS and impaired Epo production through the desensitization of the oxygen-sensing system in erythropoietin-producing cells was indicated [32]. Experimental analysis revealed that RBC in hypoxia condition show less oxygen binding affinity, probably due to a Hemoglobin conformation modification [33]. Hypoxia was also shown to promote iron release from RBCs and methemoglobin formation, which render RBCs unable to deliver oxygen to the tissues [34].

We recognize that this work has limitations. First, a study evaluating eryptosis in an elderly population found that elderly subjects have higher PS externalization than younger individuals [35]. In the present work, the HD population is significantly older than the healthy population, and therefore some of the findings presented here might have age as a contributor. A second limitation of this study is attributed to the fact that our hypoxia 
model $\left(5 \% \mathrm{O}_{2}\right)$ is much more aggressive than the oxygen levels experienced by HD patients. Therefore, further research is needed to better translate the effects of hypoxemia to CKD patients.

\section{Conclusion}

In conclusion, we found that HD-RBC have impaired RBC survival by mechanisms of eryptosis and show reduced intracellular levels of oxygen, particularly after the HD session. Also, the incubation in $5 \% \mathrm{O}_{2}$ per se promoted an increase in eryptosis in both CON-RBC and HD-RBC. In addition, S-HD decreased intracellular oxygen levels. These effects were partially inhibited by healthy serum and OAT2 inhibition. Together, these findings indicate the importance of uremic toxins in driving eryptosis and aggravating intracellular hypoxemia, possibly contributing to RBC clearance and renal anemia.

\section{Acknowledgements}

We would like to thank all patients and healthy subjects that were willing to donate blood samples to the study. We also thank the staff from the dialysis center at the Hospital Santa Casa de Curitiba, in special Dr. Ana Claudia Dambiski. Finally, the authors thank Prof. Márcia Olandoski from PUCPR, who was essential in advising the statistical analysis.

\section{Author Contributions}

Dias, G.F. performed the experiments and prepared the manuscript. Tozoni, S. S., Bohnen, G., Rodrigues, S.D., Meireles, T. and Nakao, L.S. performed the experiments and reviewed/ edited the manuscript. Pecoits-Filho, R. and Grobe. N. reviewed/edited the manuscript. Kotanko, P. and Moreno-Amaral, A.N. conceived the idea and reviewed/edited the manuscript. All authors participated in discussions during the progress of the study.

\section{Funding Sources}

This study was funded by Renal Research Institute, NY, USA. Dias, G.F. was a recipient of research scholarship from the Fundação Araucária/Coordenação de Aperfeiçoamento de Pessoal de Nível Superior-Brasil (CAPES). Bohnen, G. and Tozoni, S. S. were recipients of research scholarship from CAPES.

\section{Statement of Ethics}

The study was approved by the ethics committee of Pontifícia Universidade Católica do Paraná (registration number 1.752.213). Participants gave written informed consent before blood collection.

\section{Disclosure Statement}

Dias, G.F. has been a consultant for Renal Research Institute, NY, USA. Kotanko, P. is an employee of the Renal Research Institute, a wholly owned subsidiary of Fresenius Medical Care; he holds stock in Fresenius Medical Care. The other authors have no conflicts of interest to declare. 


\section{Cellular Physiology Cell Physiol Biochem 2021;55:449-459 \begin{tabular}{ll|l} 
and Biochemistry & $\begin{array}{l}\text { DOl: 10.33594/000000396 } \\
\text { Published online: } 14 \text { July } 2021\end{array}$ & $\begin{array}{l}\text { O } 2021 \text { The Author(s). Published by } \\
\text { Cell Physiol Biochem Press GmbH\&Co. KG }\end{array}$ \\
\cline { 2 - 3 }
\end{tabular}}

Ferreira Dias et al.: Hypoxia and Uremia in Eryptosis

\section{References}

1 Tombak A: Erythrocytes - Basis of Life. IntechOpen, 2012, pp 1-3.

2 Helms CC, Gladwin MT, Kim-Shapiro DB: Erythrocytes and vascular function: Oxygen and nitric oxide. Front Physiol 2018;9:1-9.

3 Yilmaz MI, Solak Y, Covic A, Goldsmith D, Kanbay M: Renal anemia of inflammation: The name is selfexplanatory. Blood Purif 2011;32:220-225.

4 Weiss G, Goodnough LT: Anemia of Chronic Disease. N Engl J Med 2005;352:1011-1023.

5 Ogawa T, Nitta K: Erythropoiesis-stimulating agent hyporesponsiveness in end-stage renal disease patients. Contrib Nephrol 2015;185:76-86.

6 Föller M, Lang F: Ion Transport in Eryptosis, the Suicidal Death of Erythrocytes. Front Cell Dev Biol 2020;8:1-9.

7 Bonomini M, Sirolli V, Reale M, Arduini A: Involvement of phosphatidylserine exposure in the recognition and phagocytosis of uremic erythrocytes. Am J Kidney Dis 2001 Apr;37:807-814.

8 Bonan NB, Steiner TM, Kuntsevich V, Virzì GM, Azevedo M, Nakao LS, Barreto FC, Ronco C, Thijssen S, Kotanko P, Pecoits-Filho R, Moreno-Amaral AN: Uremic Toxicity-Induced Eryptosis and Monocyte Modulation: The Erythrophagocytosis as a Novel Pathway to Renal Anemia. Blood Purif 2016;41:317-323.

9 Bonomini M, Sirolli V, Settefrati N, Dottori S, Di Liberato L, Arduini A: Increased erythrocyte phosphatidylserine exposure in chronic renal failure. J Am Soc Nephrol 1999;10:1982-1990.

10 Dias GF, Bonan NB, Steiner TM, Tozoni SS, Rodrigues S, Nakao LS, Kuntsevich V, Pecoits-Filho R, Kotanko P, Moreno-Amaral AN: Indoxyl sulfate, a uremic toxin, stimulates reactive oxygen species production and erythrocyte cell death supposedly by an organic anion transporter 2 (OAT2) and NADPH oxidase activitydependent pathways. Toxins (Basel) 2018;10:1-11.

11 Ahmed MSE, Abed M, Voelkl J, Lang F: Triggering of suicidal erythrocyte death by uremic toxin indoxyl sulfate. BMC Nephrol 2013;14:1-7.

12 Meyring-Wosten A, Zhang H, Ye X, Fuertinger DH, Chan L, Kappel F, Artemyev M, Ginsberg N, Wang Y, Thijssen S, Kotanko P: Intradialytic Hypoxemia and Clinical Outcomes in Patients on Hemodialysis. Clin J Am Soc Nephrol 2016;11:616-625.

13 Tozoni SS, Dias GF, Bohnen G, Grobe N, Pecoits-Filho R, Kotanko P, Moreno-Amaral AN: Uremia and Hypoxia Independently Induce Eryptosis and Erythrocyte Redox Imbalance. Cell Physiol Biochem 2019;53:794804.

14 Bi Y, Mathialagan S, Tylaska L, Fu M, Keefer J, Vildhede A, Costales C, Rodrigues AD, Varma MVS: Organic Anion Transporter 2 mediates hepatic uptake of tolbutamide, a Cytochrome P450 2C9 probe drug. J Pharmacol Exp Ther 2018;364:390-398.

15 Stockler-Pinto MB, Soulage CO, Borges NA, Cardozo LFMF, Dolenga CJ, Nakao LS, Pecoits-Filho R, Fouque D, Mafra D: From bench to the hemodialysis clinic: protein-bound uremic toxins modulate NF- $\kappa \mathrm{B} / \mathrm{Nrf2}$ expression. Int Urol Nephrol 2017;50:347-354.

16 Rodrigues SD, Santos SS, Meireles T, Romero N, Glorieux G, Pecoits-Filho R, Zhang DD, Nakao LS: Uremic toxins promote accumulation of oxidized protein and increased sensitivity to hydrogen peroxide in endothelial cells by impairing the autophagic flux. Biochem Biophys Res Commun 2020;523:123-129.

17 Calderón-Salinas J V., Muñoz-Reyes EG, Guerrero-Romero JF, Rodríguez-Morán M, Bracho-Riquelme RL, Carrera-Gracia MA, Quintanar-Escorza MA: Eryptosis and oxidative damage in type 2 diabetic mellitus patients with chronic kidney disease. Mol Cell Biochem 2011;357:171-179.

18 Lang E, Gatidis S, Freise NF, Bock H, Kubitz R, Lauermann C, Orth HM, Klindt C, Schuier M, Keitel V, Reich M, Liu G, Schmidt S, Xu HF, Qadri SM, Herebian D, Pandyra AA, Mayatepek E, Gulbins E, Lang F, et al.: Conjugated bilirubin triggers anemia by inducing erythrocyte death. Hepatology 2015;61:275-284.

19 Pinzón-Díaz CE, Calderón-Salinas JV, Rosas-Flores MM, Hernández G, López-Betancourt A, QuintanarEscorza MA: Eryptosis and oxidative damage in hypertensive and dyslipidemic patients. Mol Cell Biochem 2017;1-9.

20 Abed M, Artunc F, Alzoubi K, Honisch S, Baumann D, Föller M, Lang F: Suicidal erythrocyte death in endstage renal disease. J Mol Med (Berl) 2014;92:871-879.

21 Li W, Zhao Y, Fu P: Hypoxia Induced Factor in Chronic Kidney Disease: Friend or Foe? Front Med 2018;4:15 . 


\section{Cellular Physiology Cell Physiol Biochem 2021;55:449-459 \begin{tabular}{l|l|l} 
DOl: $10.33594 / 000000396$ & O 2021 The Author(s). Published by \\
and Biochemistry Published online: 14 July 2021 & Cell &
\end{tabular} \\ Ferreira Dias et al.: Hypoxia and Uremia in Eryptosis}

22 Navarrete-Opazo A, Mitchell GS: Therapeutic potential of intermittent hypoxia: a matter of dose. Am J Physiol Regul Integr Comp Physiol 2014;307:1181-1197.

23 Guedes M, Robinson BM, Obrador G, Tong A, Pisoni RL, Pecoits-Filho R: Management of Anemia in NonDialysis Chronic Kidney Disease: Current recommendations, real-world practice, and patient perspectives. Kidney360 2020;1:1-17.

24 Ma J, Dou Y, Zhang H, Thijssen S, Williams S, Kuntsevich V, Ouellet G, Wong MMY, Persiv V, Kruse A, Rosales L, Wang Y, Levin NW, Kotanko P: Correlation between Inflammatory Biomarkers and Red Blood Cell Life Span in Chronic Hemodialysis Patients. Blood Purif 2017;43:200-205.

25 Ahmed MSE, Langer H, Abed M, Voelkl J, Lang F: The uremic toxin acrolein promotes suicidal erythrocyte death. Kidney Blood Press Res 2013;37:158-167.

26 Gao C, Ji S, Dong W, Qi Y, Song W, Cui D, Shi J: Indolic Uremic Solutes Enhance Procoagulant Activity of Red Blood Cells through Phosphatidylserine Exposure and Microparticle Release. Toxins (Basel) 2015;7:43904403.

27 Deltombe 0, Glorieux G, Marzouki S, Masereeuw R, Schneditz D, Eloot S: Selective Transport of ProteinBound Uremic Toxins in Erythrocytes. Toxins (Basel) 2019;11:1-15.

28 Sirich TL, Meyer TW, Gondouin B, Brunet P, Niwa T: Protein-Bound Molecules: A Large Family With a Bad Character. Semin Nephrol2014;34:106-117.

29 Sager G, Smaglyukova N, Fuskevaag OM: The role of OAT2 (SLC22A7) in the cyclic nucleotide biokinetics of human erythrocytes. J Cell Physiol 2018;233:5972-5980.

30 Miyamoto Y, Iwao Y, Tasaki Y, Sato K, Ishima Y, Watanabe H, Kadowaki D, Maruyama T, Otagiri M: The uremic solute indoxyl sulfate acts as an antioxidant against superoxide anion radicals under normalphysiological conditions. FEBS Lett 2010;584:2816-2820.

31 Palm F, Nangaku M, Fasching A, Tanaka T, Nordquist L, Hansell P, Kawakami T, Nishijima F, Fujita T: Uremia induces abnormal oxygen consumption in tubules and aggravates chronic hypoxia of the kidney via oxidative stress. Am J Physiol Renal Physiol 2010;299:380-386.

32 Chiang CK, Tanaka T, Inagi R, Fujita T, Nangaku M: Indoxyl sulfate, a representative uremic toxin, suppresses erythropoietin production in a HIF-dependent manner. Lab Invest 2011;91:1564-1571.

33 Revin V, Grunyushkin I, Gromova N, Revina E, Abdulvwahid ASA, Solomadin I, Tychokov A, Kukina A: Effect of hypoxia on the composition and state of lipids and oxygen-transport properties of erythrocyte haemoglobin. Biotechnol Biotechnol Equip 2017;31:128-137.

34 Ciccoli L, Rossi V, Leoncini S, Signorini C, Blanco-Garcia J, Aldinucci C, Buonocore G, Comporti M: Iron release, superoxide production and binding of autologous IgG to band 3 dimers in newborn and adult erythrocytes exposed to hypoxia and hypoxia-reoxygenation. Biochim Biophys Acta 2004;1672:203-213.

35 Lupescu A, Bissinger R, Goebel T, Salker MS, Alzoubi K, Liu G, Chirigiu L, Mack AF, Qadri SM, Lang F: Enhanced suicidal erythrocyte death contributing to anemia in the elderly. Cell Physiol Biochem 2015;36:773-783. 